\title{
Signatures of avoided energy-level crossings in entanglement indicators obtained from quantum tomograms
}

\author{
B. Sharmila ${ }^{1}$, S. Lakshmibala ${ }^{1}$ and V. Balakrishnan ${ }^{1}$ \\ ${ }^{1}$ Department of Physics, Indian Institute of Technology Madras, Chennai 600036, \\ India. \\ E-mail: sharmilab@physics.iitm.ac.in
}

29 June 2020

\begin{abstract}
Extensive theoretical and experimental investigations on multipartite systems close to an avoided energy-level crossing reveal interesting features such as the extremisation of entanglement. Conventionally, the estimation of entanglement directly from experimental observation involves either one of two approaches: Uncertainty-relation-based estimation that captures the linear correlation between relevant observables, or rigorous but error-prone quantum state reconstruction on tomograms obtained from homodyne measurements. We investigate the behaviour, close to avoided crossings, of entanglement indicators that can be calculated directly from a numerically-generated tomogram. The systems we study are two generic bipartite continuous-variable systems: a Bose-Einstein condensate trapped in a doublewell potential, and a multi-level atom interacting with a radiation field. We also consider a multipartite hybrid quantum system of superconducting qubits interacting with microwave photons. We carry out a quantitative comparison of the indicators with a standard measure of entanglement, the subsystem von Neumann entropy (SVNE). It is shown that the indicators that capture the nonlinear correlation between relevant subsystem observables are in excellent agreement with the SVNE.
\end{abstract}

Keywords: Energy-level crossings, Quantum entanglement, Tomograms, Tomographic entanglement indicator

Submitted to: J. Phys. B: At. Mol. Phys. 


\section{Introduction}

The measurement of any observable in a quantum mechanical system yields a histogram of the state of the system in the basis of that observable. In particular, in the context of quantum optics, measurements of a judiciously chosen quorum of field observables yield a set of histograms (the optical tomogram) from which the density matrix is reconstructed. The latter is needed in standard procedures for estimating the extent of entanglement between the subsystems of bipartite or multipartite systems. A standard measure of the entanglement between the two subsystems of a bipartite system is the subsystem von Neumann entropy $\xi_{\mathrm{SVNE}}=-\operatorname{Tr}\left(\rho \log _{2} \rho\right)$, where $\rho$ is the density matrix of either one of the subsystems [1]. In the case of continuous-variable (CV) quantum systems, an infinite set of histograms is required, in principle, in order to obtain complete information about the density matrix. In practice, however, only a finite set of histograms (corresponding to measurement of a finite set of observables) can be obtained. As state reconstruction from tomograms [2] typically involves error-prone statistical techniques such as maximum likelihood estimates, it is preferable to assess the extent of entanglement directly from tomograms, circumventing detailed state reconstruction.

In earlier work [3] we have proposed a tomographic entanglement indicator $\xi_{\text {TEI }}$ based on mutual information, that is obtained directly from the relevant tomograms. We have tested its efficacy in bipartite CV systems evolving unitarily under nonlinear Hamiltonians, by comparing it both with $\xi_{\text {SVNE }}$ and with an entanglement indicator $\xi_{\text {IPR }}$ based on inverse participation ratios [4]. (The participation ratio is a measure of delocalization in a given basis.) It has been shown that $\xi_{\text {TEI }}$ and $\xi_{\text {IPR }}$ capture the gross features of entanglement dynamics. A time-series analysis of the difference $\left|\xi_{\text {TEI }}-\xi_{\text {SVNE }}\right|$ was used to quantify the deviation of $\xi_{\text {TEI }}$ from $\xi_{\text {SVNE }}$ as a function of time. Although this is sensitive to the specific choice of initial state and the strength of the nonlinearity in the Hamiltonian, it has been shown that $\xi_{\text {TEI }}$ is a reasonably good indicator of entanglement in general. In multipartite hybrid quantum (HQ) systems comprising two-level atoms interacting with radiation fields, too, $\xi_{\text {TEI }}$ turns out [5] to be a good estimator of quantum correlations in both the field and the atomic subsystems. (In the atom sector, $\xi_{\text {TEI }}$ is extracted directly from the corresponding qubit tomograms).

Several indicators of correlations between different parts of a classical system have been used extensively in various applications such as automated image processing. These correlators are obtained from classical tomograms. Their definitions, however, are not intrinsically classical in nature, and it is worth examining their applicability in quantum contexts. Since correlations are inherently present in entangled states of quantum systems, a natural question that arises is whether the performance of entanglement quantifiers obtained from these correlators is comparable to that of standard indicators such as $\xi_{\mathrm{SVNE}}$.

We examine quantum systems where the spacings between energy levels change significantly with changes in the parameters, with two or more levels moving close to each other for specific values of the parameters and then moving away as these values change. 
We will henceforth refer to this feature as avoided energy-level crossing. Extensive studies [6] 8] have established that entanglement (as measured by standard indicators such as $\left.\xi_{\mathrm{SVNE}}\right)$ is generically at an extremum at an avoided crossing. Typically, the energy spectrum and the spacing between the energy levels depend on the strengths of the nonlinearity and the coupling between subsystems. With changes in the values of these parameters, the spacing between adjacent levels can decrease, and even tend to zero, resulting in an energy-level crossing. According to the von Neumann-Wigner nocrossing theorem, energy levels within a multiplet generically avoid crossing, provided only one of the parameters is varied in the Hamiltonian governing the system. In this paper, we investigate how effectively some of these entanglement indicators mimic the behaviour of $\xi_{\mathrm{SVNE}}$ close to avoided crossings.

Energy-level crossings display other interesting features. Since they affect the level spacings and their probability distribution [9], they are also important from the point of view of non-integrability and quantum chaos (see, for instance, [10]). In addition, avoided crossings point to phase transitions which trigger a change in the quantum correlations in the system [6, 7, 11, 12]. This aspect has been investigated extensively both theoretically and in experiments [13 16].

We examine two experimentally relevant bipartite CV systems: a Bose-Einstein condensate (BEC) in a double-well trap [17], and a multilevel atom interacting with a radiation field [18. We also investigate a multipartite $\mathrm{HQ}$ system [19, 20] that is effectively described by the Tavis-Cummings model [21]. The rest of this paper is organized as follows: In the next section we introduce the entanglement indicators to be employed. In section 3, we investigate how these indicators behave close to avoided crossings in the two bipartite CV models mentioned above. In section 4, we extend our analysis to the multipartite HQ model. Concluding remarks are made in section 5 .

\section{Entanglement indicators from tomograms}

We first consider generic CV systems. A typical example of a bipartite CV system is two coupled oscillators (equivalently, a single-mode radiation field interacting with a multilevel atom modelled as an oscillator). The tomogram is obtained from the quorum of observables that contain complete information about the state. These observables are represented by the rotated quadrature operators

$$
\mathbb{X}_{\theta_{\mathrm{A}}}=\left(a e^{-i \theta} \mathrm{A}+a^{\dagger} e^{i \theta} \mathrm{A}\right) / \sqrt{2}, \quad \mathbb{X}_{\theta_{\mathrm{B}}}=\left(b e^{-i \theta} \mathrm{B}+b^{\dagger} e^{i \theta} \mathrm{B}\right) / \sqrt{2} .
$$

Here $0 \leqslant \theta_{\mathrm{A}}, \theta_{\mathrm{B}}<\pi$, and $\left(a, a^{\dagger}\right)$ [respectively, $\left(b, b^{\dagger}\right)$ ] are the oscillator annihilation and creation operators corresponding to the two subsystems A and B. The bipartite tomogram is given by

$$
w\left(X_{\theta_{\mathrm{A}}}, \theta_{\mathrm{A}} ; X_{\theta_{\mathrm{B}}}, \theta_{\mathrm{B}}\right)=\left\langle X_{\theta_{\mathrm{A}}}, \theta_{\mathrm{A}} ; X_{\theta_{\mathrm{B}}}, \theta_{\mathrm{B}}\left|\rho_{\mathrm{AB}}\right| X_{\theta_{\mathrm{A}}}, \theta_{\mathrm{A}} ; X_{\theta_{\mathrm{B}}}, \theta_{\mathrm{B}}\right\rangle,
$$

where $\rho_{\mathrm{AB}}$ denotes the bipartite density matrix. Here $\mathbb{X}_{\theta_{i}}\left|X_{\theta_{i}}, \theta_{i}\right\rangle=X_{\theta_{i}}\left|X_{\theta_{i}}, \theta_{i}\right\rangle$ $(i=\mathrm{A}, \mathrm{B})$, and the product basis state $\left|X_{\theta_{\mathrm{A}}}, \theta_{\mathrm{A}}\right\rangle \otimes\left|X_{\theta_{\mathrm{B}}}, \theta_{\mathrm{B}}\right\rangle$ is written as 
$\left|X_{\theta_{\mathrm{A}}}, \theta_{\mathrm{A}} ; X_{\theta_{\mathrm{B}}}, \theta_{\mathrm{B}}\right\rangle$. The normalization condition is given by

$$
\int_{-\infty}^{\infty} \mathrm{d} X_{\theta_{\mathrm{A}}} \int_{-\infty}^{\infty} \mathrm{d} X_{\theta_{\mathrm{B}}} w\left(X_{\theta_{\mathrm{A}}}, \theta_{\mathrm{A}} ; X_{\theta_{\mathrm{B}}}, \theta_{\mathrm{B}}\right)=1
$$

for each $\theta_{\mathrm{A}}$ and $\theta_{\mathrm{B}}$. The reduced tomogram for subsystem $\mathrm{A}$ is

$$
\begin{aligned}
w_{\mathrm{A}}\left(X_{\theta_{\mathrm{A}}}, \theta_{\mathrm{A}}\right) & =\int_{-\infty}^{\infty} \mathrm{d} X_{\theta_{\mathrm{B}}} w\left(X_{\theta_{\mathrm{A}}}, \theta_{\mathrm{A}} ; X_{\theta_{\mathrm{B}}}, \theta_{\mathrm{B}}\right) \\
& =\left\langle X_{\theta_{\mathrm{A}}}, \theta_{\mathrm{A}}\left|\rho_{\mathrm{A}}\right| X_{\theta_{\mathrm{A}}}, \theta_{\mathrm{A}}\right\rangle,
\end{aligned}
$$

where $\rho_{\mathrm{A}}=\operatorname{Tr}_{\mathrm{B}}\left(\rho_{\mathrm{AB}}\right)$ is the corresponding reduced density matrix. A similar definition holds for subsystem B. In order to estimate the degree of correlation between the subsystems, we use the following tomographic entropies. The bipartite tomographic entropy is given by

$$
\begin{aligned}
S\left(\theta_{\mathrm{A}}, \theta_{\mathrm{B}}\right)=- & \int_{-\infty}^{\infty} \mathrm{d} X_{\theta_{\mathrm{A}}} \int_{-\infty}^{\infty} \mathrm{d} X_{\theta_{\mathrm{B}}} w\left(X_{\theta_{\mathrm{A}}}, \theta_{\mathrm{A}} ; X_{\theta_{\mathrm{B}}}, \theta_{\mathrm{B}}\right) \times \\
& \log _{2} w\left(X_{\theta_{\mathrm{A}}}, \theta_{\mathrm{A}} ; X_{\theta_{\mathrm{B}}}, \theta_{\mathrm{B}}\right) .
\end{aligned}
$$

The subsystem tomographic entropy is

$$
S\left(\theta_{i}\right)=-\int_{-\infty}^{\infty} \mathrm{d} X_{\theta_{i}} w_{i}\left(X_{\theta_{i}}, \theta_{i}\right) \log _{2}\left[w_{i}\left(X_{\theta_{i}}, \theta_{i}\right)\right] \quad(i=\mathrm{A}, \mathrm{B}) .
$$

Some of the correlators that we examine in this paper are obtained from a section of the tomogram corresponding to specific values of $\theta_{\mathrm{A}}$ and $\theta_{\mathrm{B}}$. The efficacy of such a correlator as a measure of entanglement is therefore sensitive to the choice of the tomographic section. We now define these correlators, and the corresponding entanglement indicators.

The mutual information $\varepsilon_{\text {TEI }}\left(\theta_{\mathrm{A}}, \theta_{\mathrm{B}}\right)$ which we get from the tomogram of a quantum system can carry signatures of entanglement. This quantity is expressed in terms of the tomographic entropies defined above as

$$
\varepsilon_{\mathrm{TEI}}\left(\theta_{\mathrm{A}}, \theta_{\mathrm{B}}\right)=S\left(\theta_{\mathrm{A}}\right)+S\left(\theta_{\mathrm{B}}\right)-S\left(\theta_{\mathrm{A}}, \theta_{\mathrm{B}}\right) .
$$

Indicators based on the inverse participation ratio (IPR) are also found to be good candidates for estimating the extent of entanglement [4,22]. The IPR corresponding to a bipartite system in the basis of the rotated quadrature operators is defined as

$$
\eta_{\mathrm{AB}}\left(\theta_{\mathrm{A}}, \theta_{\mathrm{B}}\right)=\int_{-\infty}^{\infty} \mathrm{d} X_{\theta_{\mathrm{A}}} \int_{-\infty}^{\infty} \mathrm{d} X_{\theta_{\mathrm{B}}}\left[w\left(X_{\theta_{\mathrm{A}}}, \theta_{\mathrm{A}} ; X_{\theta_{\mathrm{B}}}, \theta_{\mathrm{B}}\right)\right]^{2} .
$$

The IPR for each subsystem is given by

$$
\eta_{i}\left(\theta_{i}\right)=\int_{-\infty}^{\infty} \mathrm{d} X_{\theta_{i}}\left[w_{i}\left(X_{\theta_{i}}, \theta_{i}\right)\right]^{2} \quad(i=\mathrm{A}, \mathrm{B}) .
$$

The entanglement indicator in this case is given by

$$
\varepsilon_{\mathrm{IPR}}\left(\theta_{\mathrm{A}}, \theta_{\mathrm{B}}\right)=1+\eta_{\mathrm{AB}}\left(\theta_{\mathrm{A}}, \theta_{\mathrm{B}}\right)-\eta_{\mathrm{A}}\left(\theta_{\mathrm{A}}\right)-\eta_{\mathrm{B}}\left(\theta_{\mathrm{B}}\right) .
$$


Apart from these, we have examined two other correlators which are familiar in the context of classical tomograms. The first of these is the Pearson correlation coefficient [23] between two random variables $X$ and $Y$, given by

$$
\operatorname{PCC}(X, Y)=\frac{\operatorname{Cov}(X, Y)}{\sigma_{\mathrm{X}} \sigma_{\mathrm{Y}}} .
$$

Here $\sigma_{\mathrm{X}}, \sigma_{\mathrm{Y}}$ are the standard deviations of $X$ and $Y$ respectively, and $\operatorname{Cov}(X, Y)$ is their covariance. Of direct relevance to us is $\operatorname{PCC}\left(X_{\theta_{\mathrm{A}}}, X_{\theta_{\mathrm{B}}}\right)$ calculated for fixed values of $\theta_{\mathrm{A}}$ and $\theta_{\mathrm{B}}$. Since the quantifier of entanglement between two subsystems must be nonnegative, a simple definition of the entanglement indicator in this case would be

$$
\varepsilon_{\mathrm{PCC}}\left(\theta_{\mathrm{A}}, \theta_{\mathrm{B}}\right)=\left|\mathrm{PCC}\left(X_{\theta_{\mathrm{A}}}, X_{\theta_{\mathrm{B}}}\right)\right| \text {. }
$$

This indicator captures the effect of linear correlations. Our motivation for assessing this indicator arises from the fact that, in recent experiments on generating and testing the extent of entanglement in CV systems, the variances of suitably chosen conjugate observables and the corresponding standard quantum limit alone are used [24]. We reiterate that these merely capture the extent of linear correlations between two states.

The second indicator (to be denoted by $\varepsilon_{\mathrm{BD}}$ ) that we introduce and use is arrived at as follows. In probability theory, the mutual information [25] between two continuous random variables $X$ and $Y$ can be expressed in terms of the Kullback-Leibler divergence $D_{\mathrm{KL}}$ [26] between their joint probability density $p_{X Y}(x, y)$ and the product of the corresponding marginal densities $p_{X}(x)=\int p_{X Y}(x, y) d y$ and $p_{Y}(y)=\int p_{X Y}(x, y) d x$, as 27

$$
D_{\mathrm{KL}}\left[p_{X Y}: p_{X} p_{Y}\right]=\int d x \int d y p_{X Y}(x, y) \log _{2} \frac{p_{X Y}(x, y)}{p_{X}(x) p_{Y}(y)},
$$

The quantity $\varepsilon_{\mathrm{TEI}}\left(\theta_{\mathrm{A}}, \theta_{\mathrm{B}}\right)$ defined in Eq. (77) is precisely the mutual information in the case of optical tomograms (which are continuous probability distributions):

$$
\varepsilon_{\mathrm{TEI}}\left(\theta_{\mathrm{A}}, \theta_{\mathrm{B}}\right)=D_{\mathrm{KL}}\left[w\left(X_{\theta_{\mathrm{A}}}, \theta_{\mathrm{A}} ; X_{\theta_{\mathrm{B}}}, \theta_{\mathrm{B}}\right): w_{\mathrm{A}}\left(X_{\theta_{\mathrm{A}}}, \theta\right) w_{\mathrm{B}}\left(X_{\theta_{\mathrm{B}}}, \theta_{\mathrm{B}}\right)\right] .
$$

A simpler alternative for our purposes is provided by the Bhattacharyya distance $D_{\mathrm{B}}[28]$ between $p_{X Y}$ and $p_{X} p_{Y}$, defined as

$$
D_{\mathrm{B}}\left[p_{X Y}: p_{X} p_{Y}\right]=-\log _{2}\left\{\int d x \int d y\left[p_{x y}(x, y) p_{X}(x) p_{Y}(y)\right]^{1 / 2}\right\} .
$$

Using Jensen's inequality, it is easily shown that $D_{\mathrm{B}} \leqslant \frac{1}{2} D_{\mathrm{KL}} . \quad D_{\mathrm{B}}$ thus gives us an approximate estimate (that is an underestimate) of the mutual information. Based on this quantity, we have an entanglement indicator that is the analogue of Eq. (14), namely,

$$
\varepsilon_{\mathrm{BD}}\left(\theta_{\mathrm{A}}, \theta_{\mathrm{B}}\right)=D_{\mathrm{B}}\left[w\left(X_{\theta_{\mathrm{A}}}, \theta_{\mathrm{A}} ; X_{\theta_{\mathrm{B}}}, \theta_{\mathrm{B}}\right): w_{\mathrm{A}}\left(X_{\theta_{\mathrm{A}}}, \theta\right) w_{\mathrm{B}}\left(X_{\theta_{\mathrm{B}}}, \theta_{\mathrm{B}}\right)\right]
$$

The dependence on $\theta_{\mathrm{A}}$ and $\theta_{\mathrm{B}}$ of each of the foregoing entanglement indicators $\varepsilon$ is removed by averaging over a representative set of values of those variables. We denote the corresponding averaged value by $\xi$. In the context of bipartite CV models, we have shown in earlier work [3, 4] that averaging $\varepsilon_{\text {TEI }}\left(\theta_{\mathrm{A}}, \theta_{\mathrm{B}}\right)$ over 25 different values 
of $\left(\theta_{\mathrm{A}}, \theta_{\mathrm{B}}\right)$ selected at equal intervals in the range $[0, \pi)$ yields a reliable entanglement indicator $\xi_{\mathrm{TEI}}$. A similar averaging of each of the quantities $\varepsilon_{\mathrm{IPR}}, \varepsilon_{\mathrm{PCC}}$ and $\varepsilon_{\mathrm{BD}}$ yields $\xi_{\mathrm{IPR}}, \xi_{\mathrm{PCC}}$ and $\xi_{\mathrm{BD}}$, respectively.

Next, we turn to hybrid systems of field-atom interactions. For a two-level atom with ground state $|g\rangle$ and excited state $|e\rangle$, the quorum of observables is [29]

$$
\begin{gathered}
\sigma_{x}=\frac{1}{2}(|e\rangle\langle g|+| g\rangle\langle e|), \sigma_{y}=\frac{1}{2} i(|g\rangle\langle e|-| e\rangle\langle g|), \\
\sigma_{z}=\frac{1}{2}(|e\rangle\langle e|-| g\rangle\langle g|),
\end{gathered}
$$

where $\sigma_{i}$ is a Pauli matrix. Let $\sigma_{z}|m\rangle=m|m\rangle$. Then $U(\vartheta, \varphi)|m\rangle=|\vartheta, \varphi, m\rangle$, where $U(\vartheta, \varphi)$ is a general $\mathrm{SU}(2)$ transformation parametrized by $(\vartheta, \varphi)$. Denoting $(\vartheta, \varphi)$ by the unit vector $\mathbf{n}$, the qubit tomogram is given by

$$
w(\mathbf{n}, m)=\left\langle\mathbf{n}, m\left|\rho_{\mathrm{S}}\right| \mathbf{n}, m\right\rangle
$$

where $\rho_{\mathrm{S}}$ is the qubit density matrix. Corresponding to each value of $\mathbf{n}$ there exists a complete basis set. The atomic tomograms are obtained from these, and the corresponding entanglement properties are quantified using appropriate adaptations of the indicators described above. The extension of the foregoing to the multipartite case is straightforward [30], and the tomograms obtained can be examined on similar lines.

\section{Avoided energy-level crossings in bipartite CV models}

\subsection{The double-well BEC model}

The effective Hamiltonian for the system and its diagonalisation are as follows [17]. Setting $\hbar=1$,

$$
H_{\mathrm{BEC}}=\omega_{0} N_{\mathrm{tot}}+\omega_{1}\left(a^{\dagger} a-b^{\dagger} b\right)+U N_{\mathrm{tot}}^{2}-\lambda\left(a^{\dagger} b+a b^{\dagger}\right) .
$$

Here, $\left(a, a^{\dagger}\right)$ and $\left(b, b^{\dagger}\right)$ are the respective boson annihilation and creation operators of the atoms in wells $\mathrm{A}$ and B (the two subsystems), and $N_{\text {tot }}=\left(a^{\dagger} a+b^{\dagger} b\right)$. $U$ is the strength of nonlinear interactions between atoms within each well, and also between the two wells. $U>0$, ensuring that the energy spectrum is bounded from below. $\lambda$ is the linear interaction strength, while $\omega_{1}$ is the strength of the population imbalance between the two wells. The Hamiltonian is diagonalised by the unitary transformation $V=e^{\kappa\left(a^{\dagger} b-b^{\dagger} a\right) / 2}$ where $\kappa=\tan ^{-1}\left(\lambda / \omega_{1}\right)$, to yield

$$
V^{\dagger} H_{\mathrm{BEC}} V=\widetilde{H}_{\mathrm{BEC}}=\omega_{0} N_{\text {tot }}+\lambda_{1}\left(a^{\dagger} a-b^{\dagger} b\right)+U N_{\text {tot }}^{2},
$$

with $\lambda_{1}=\left(\lambda^{2}+\omega_{1}^{2}\right)^{1 / 2}$. $\widetilde{H}_{\mathrm{BEC}}$ and $N_{\text {tot }}$ commute with each other. Their common eigenstates are the product states $|k\rangle \otimes|N-k\rangle \equiv|k, N-k\rangle$. Here $N=0,1,2, \ldots$ is the eigenvalue of $N_{\text {tot }}$, and $|k\rangle$ is a boson number state, with $k$ running from 0 to $N$ for a given $N$. The eigenstates and eigenvalues of $H_{\mathrm{BEC}}$ are given by

$$
\left|\psi_{N, k}\right\rangle=V|k, N-k\rangle
$$

and

$$
E(N, k)=\omega_{0} N+\lambda_{1}(2 k-N)+U N^{2}
$$



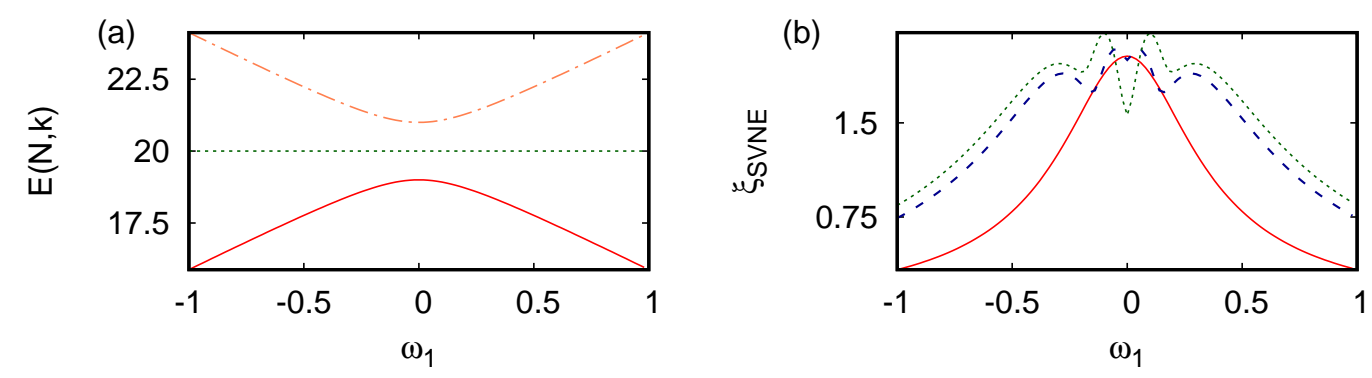

Figure 1. (a) $E(N, k)$ vs. $\omega_{1}$ for $N=4$ and $k=0,2,4$ in the BEC model. (b) $\xi_{\text {SVNE }}$ vs. $\omega_{1}$ for $N=4, k=0,1,2$. The curves correspond to $k=0$ (red solid), 1 (blue dashed), 2 (green dotted) and 4 (orange dot-dashed). $\lambda=0.25$.

For numerical analysis we set $\omega_{0}=1, U=1$.

In figure 1(a), $E(N=4, k)$ is plotted against $\omega_{1}$ for $k=0,2,4$, with $\lambda=0.25$. $E(N, N-k)$ is the reflection of $E(N, k)$ about the value $\omega_{0} N+U N^{2}$. Avoided energylevel crossings are seen at $\omega_{1}=0$. In order to set the reference level for the extent of entanglement between the two wells, we compute $\xi_{\mathrm{SVNE}}=-\operatorname{Tr}\left(\rho_{\mathrm{A}} \log _{2} \rho_{\mathrm{A}}\right)$, where $\rho_{\mathrm{A}}$ is the reduced density matrix of the subsystem $\mathrm{A}$. ( $\xi_{\mathrm{SVNE}}$ is also equal to $-\operatorname{Tr}\left(\rho_{\mathrm{B}} \log _{2} \rho_{\mathrm{B}}\right)$, since $\left|\psi_{N, k}\right\rangle$ is a bipartite pure state.) Plots of $\xi_{\mathrm{SVNE}}$ corresponding to the state $\left|\psi_{4, k}\right\rangle$ for $k=0,1,2$ are shown in figure 1(b). The states $\left|\psi_{4,3}\right\rangle$ and $\left|\psi_{4,1}\right\rangle$ have the same $\xi_{\mathrm{SVNE}}$, (as do the states $\left|\psi_{4,4}\right\rangle$ and $\left|\psi_{4,0}\right\rangle$ ), owing to the $k \leftrightarrow N-k$ symmetry. It is evident that there is a significant extent of entanglement close to the avoided crossing, and $\omega_{1}=0$ is marked by a local maximum or minimum in $\xi_{\mathrm{SVNE}}$.

Figure 2 depicts $\theta_{\mathrm{A}}=0, \theta_{\mathrm{B}}=\frac{1}{2} \pi$ sections of the tomograms corresponding to the states $\left|\psi_{4, k}\right\rangle$ for $k=0,1,2$ and $\omega_{1}=0,0.1,1$. It is clear that, for a given value of $\omega_{1}$, the qualitative features of the tomograms are altered considerably as $k$ is varied. The patterns in the tomograms also reveal nonlinear correlations between the quadrature variables $X_{\theta_{\mathrm{A}}}$ and $X_{\theta_{\mathrm{B}}}$ (top panel). For instance, the tomogram slice on the top right shows a probability distribution that is essentially unimodal and symmetric about the origin with the annular structures diminished in magnitude. It is clear that this case is less correlated than the tomogram in the top left corner. This conforms to the observed trend in the extent of entanglement (compare $\xi_{\mathrm{SVNE}}$ corresponding to $k=0$ and $k=2$ at $\omega_{1}=0$ in figure 1 (b)). Again, in the bottom panel of the figure, the sub-structures in the patterns increase with increasing $k$, signifying a higher degree of nonlinear correlation. This is in consonance with the trend in the entanglement at $\omega_{1}=1$ (figure 1 (b)). We therefore expect $\varepsilon_{\mathrm{TEI}}$ and its averaged version $\xi_{\mathrm{TEI}}$ to be much better entanglement indicators than $\varepsilon_{\mathrm{PCC}}$ and $\xi_{\mathrm{PCC}}$. We also mention here that the current experimental techniques of testing $\mathrm{CV}$ entanglement based on the variances and covariances of suitably chosen observables [24] are not as effective as calculating nonlinear correlators, for the same reason.

Our detailed investigations reveal that $\xi_{\mathrm{TEI}}$ and $\xi_{\mathrm{IPR}}$ follow the trends in $\xi_{\mathrm{SVNE}}$ reasonably well for generic eigenstates of $H_{\mathrm{BEC}}$. This is illustrated in figure 3, which 

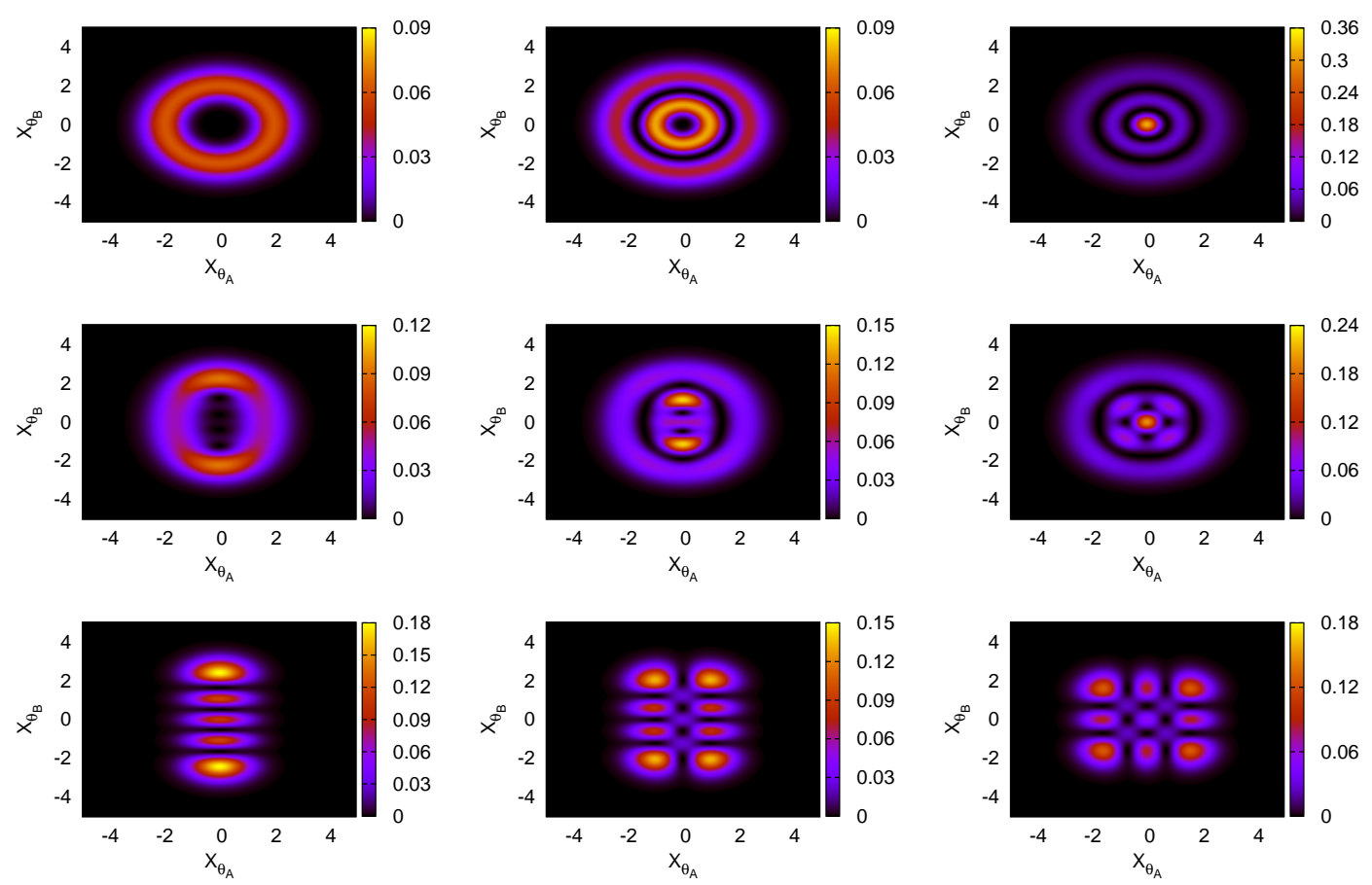

Figure 2. $\theta_{\mathrm{A}}=0, \theta_{\mathrm{B}}=\frac{1}{2} \pi$ slice of the tomogram for $N=4$ in the BEC model. Left to right, $k=0,1$ and 2 . Top to bottom, $\omega_{1}=0,0.1$ and 1 .

shows plots of these indicators as functions of $\omega_{1}$. Apart from examining the suitability of $\varepsilon_{\mathrm{PCC}}$ as an entanglement indicator, we have also checked for the extent of linear correlation between any two indicators based on the corresponding PCC, as follows. We have obtained 100 values each of $\xi_{\mathrm{TEI}}$ and $\xi_{\mathrm{SVNE}}$ for different values of $\omega_{1}$ in the range $(-1,1)$ in steps of 0.02 . Treating the two sets of values as two sets of random numbers, we obtain the PCC between them, as defined in Eq. (11). The PCC between $\xi_{\text {TEI }}$ and $\xi_{\mathrm{SVNE}}$ (respectively, $\xi_{\mathrm{IPR}}$ and $\xi_{\mathrm{SVNE}}$ ) estimates the extent of linear correlation between the two indicators, and is found to be 0.97 (resp., 0.99) in the case shown in figure 3 corresponding to $\left|\psi_{4,2}\right\rangle$. (In general, the PCC ranges from 1 for complete correlation, to -1 for maximal anti-correlation. Its vanishing indicates the absence of linear correlation).

Figure 4 shows the PCC between $\xi_{\mathrm{SVNE}}$ and various indicators, for the eigenstates $\left|\psi_{4, k}\right\rangle$ where $k=0,1,2,3,4$. From figure 4(a), we see that $\xi_{\text {IPR }}, \xi_{\text {TEI }}$ and $\xi_{\text {BD }}$ are very good entanglement indicators. We have also found that all these indicators improve with increasing $N$. The performance of the $\varepsilon$-indicators depends, of course, on the specific choice of the tomographic section. For instance, $\varepsilon_{\mathrm{TEI}}$ and $\varepsilon_{\mathrm{BD}}$ perform marginally better for the slice $\theta_{\mathrm{A}}=0, \theta_{\mathrm{B}}=0$ than for the slice $\theta_{\mathrm{A}}=0, \theta_{\mathrm{B}}=\frac{1}{2} \pi$. It is also evident that $\xi_{\mathrm{PCC}}$ does not fare as well as the other indicators. This is to be expected, since $\xi_{\mathrm{PCC}}$ only captures linear correlations, as already emphasised.

We have verified that the sensitivity of all the indicators decreases with an increase in $\lambda$, the strength of the coupling between the two subsystems (as in Eq. 19). $\xi_{\text {IPR }}$, 


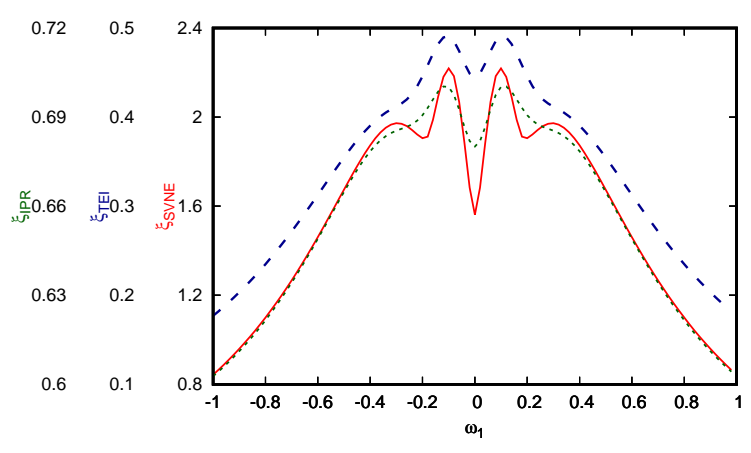

Figure 3. $\xi_{\mathrm{SVNE}}$ (red solid line), $\xi_{\mathrm{TEI}}\left(\right.$ blue dashed line) and $\xi_{\mathrm{IPR}}$ (green dotted line) vs. $\omega_{1}$, for the state $\left|\psi_{4,2}\right\rangle$ in the BEC model.
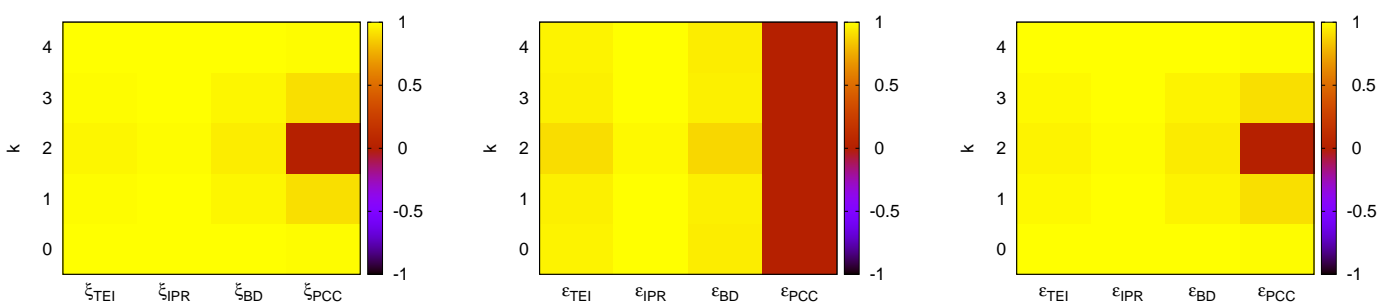

Figure 4. Correlation of $\xi_{\mathrm{SVNE}}$ with $\xi$-indicators (left), with $\varepsilon$-indicators for the slice $\theta_{\mathrm{A}}=0, \theta_{\mathrm{B}}=\frac{1}{2} \pi$ (centre), and with $\varepsilon$-indicators for the slice $\theta_{\mathrm{A}}=0, \theta_{\mathrm{B}}=0$ (right), for the eigenstates $\left|\psi_{4, k}\right\rangle, 0 \leqslant k \leqslant 4$ in the BEC model.

however, remains closer to $\xi_{\mathrm{SVNE}}$ than the other indicators. This fact is consistent with inferences drawn from our earlier work [4] about the relation between the Hamming distance [31] and the efficacy of $\xi_{\text {IPR }}$. We recall that the Hamming distance between two bipartite qudits $\left|u_{1}\right\rangle \otimes\left|u_{2}\right\rangle$ and $\left|v_{1}\right\rangle \otimes\left|v_{2}\right\rangle$ attains its maximum value of 2 when $\left\langle u_{1} \mid v_{1}\right\rangle=0$ and $\left\langle u_{2} \mid v_{2}\right\rangle=0$. A straightforward extension to CV systems implies that the Hamming distance between $\left|k_{1}, N-k_{1}\right\rangle$ and $\left|k_{2}, N-k_{2}\right\rangle$ is 2 (so that these states are Hamminguncorrelated), if $k_{1} \neq k_{2}$. Participation ratios are valid measures of entanglement for superpositions of Hamming-uncorrelated states in spin systems [22]. We have demonstrated in our earlier work that $\xi_{\text {IPR }}$ effectively mimics standard measures of entanglement in CV systems as well. In the present instance, the eigenstates $\left|\psi_{N, k}\right\rangle$ are superpositions of the states $\{|j, N-j\rangle\}$ which are Hamming-uncorrelated for different values of $j$. This is the reason for the usefulness of $\xi_{\text {IPR }}$ as an entanglement indicator even for larger values of $\lambda$.

We now proceed to examine quantitatively the efficacy of the entanglement indicators as functions of $\lambda$. For numerical computation we have set $\omega_{1}=0.25$. Consider, as an illustration, plots of the eigenvalues $E(4, k)(k=0,2,4)$ as functions of $\lambda$. These plots are exactly the same as those in figure 1(a), with $\omega_{1}$ replaced by $\lambda$ on the horizontal axis, since $E(N, k)$ only depends on the parameters $\omega_{1}$ and $\lambda$ in the symmetric combination $\lambda_{1}=\left(\lambda^{2}+\omega_{1}^{2}\right)^{1 / 2}$. The avoided crossing of energy levels now occurs at $\lambda=0$. But this symmetry between $\omega_{1}$ and $\lambda$ does not extend to the unitary 


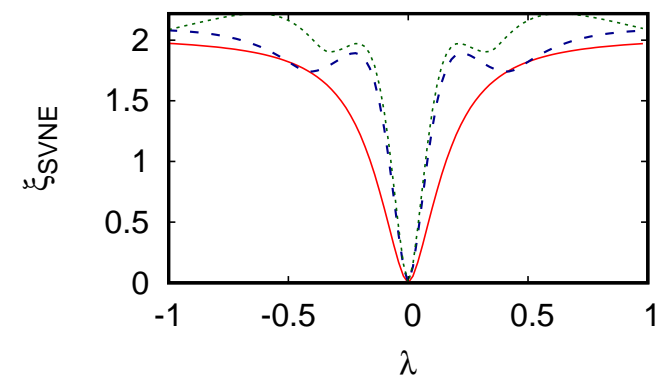

Figure 5. $\xi_{\mathrm{SVNE}}$ vs. $\lambda$ for $N=4, k=0,1,2$, for the BEC model. The curves correspond to $k=0$ (red solid), 1 (blue dashed) and 2 (green dotted). $\omega_{1}=0.25$.

transformation $V$, and hence to the eigenstates of $H_{\mathrm{BEC}}$. (Recall that $V$ involves the parameter $\kappa=\tan ^{-1}\left(\lambda / \omega_{1}\right)$.) When $\lambda=0$ there is no linear interaction between the two modes. $V$ then reduces to the identity operator, and $H_{\mathrm{BEC}}$ is diagonal in the basis $\{|k, N-k\rangle\}$. We therefore expect the entanglement to vanish at the avoided crossing. This is borne out in figure 5 in which $\xi_{\mathrm{SVNE}}$ for the state $|\psi(4, k)\rangle$ is plotted for different values of $k$. As before, it suffices to depict the cases $k=0,1$ and 2 because of the $k \leftrightarrow N-k$ symmetry. We observe that, in the case $k=0$, while there is a minimum in $\xi_{\mathrm{SVNE}}$ at $\lambda=0$, there is a maximum in this quantity at $\omega_{1}=0$ (figure 1(b)).

We have also calculated the PCC between various indicators and $\xi_{\text {SVNE }}$ for the set of states $\left|\psi_{4, k}\right\rangle, 0 \leqslant k \leqslant 4$, using 100 values of each of the $\xi$-indicators calculated for each $\lambda$ in the range $[-1,1]$ with a step size of 0.02 . The results are very similar to those already found (see figure 4) using $\omega_{1}$ as the variable parameter instead of $\lambda$.

\subsection{Atom-field interaction model}

We turn next to the case of a multi-level atom (modelled by an anharmonic oscillator) that is linearly coupled with strength $g$ to a radiation field of frequency $\omega_{\mathrm{f}}$. The effective Hamiltonian (setting $\hbar=1$ ) is given by [18]

$$
H_{\mathrm{AF}}=\omega_{\mathrm{f}} a^{\dagger} a+\omega_{\mathrm{a}} b^{\dagger} b+\gamma b^{\dagger 2} b^{2}+g\left(a^{\dagger} b+a b^{\dagger}\right) .
$$

$\omega_{\mathrm{a}}$ and $\gamma$ (>0 for stability) are constants. $\left(a, a^{\dagger}\right)$ and $\left(b, b^{\dagger}\right)$ are the annihilation and creation operators for the field mode and the oscillator mode, respectively. As before, $N_{\text {tot }}=a^{\dagger} a+b^{\dagger} b$ and $\left[H_{\mathrm{AF}}, N_{\text {tot }}\right]=0$. As in the BEC model of the preceding section, the eigenvalues $E_{\mathrm{AF}}(N, k)$ and the common eigenstates $\left|\phi_{N, k}\right\rangle$ of these two operators are labelled by $N=0,1, \ldots$ (the eigenvalue of $\left.N_{\text {tot }}\right)$ and, within each $(N+1)$-dimensional subspace for a given $N$, by the index $k$ that runs from 0 to $N$.

We find $\left|\phi_{N, k}\right\rangle$ and $E_{\mathrm{AF}}(N, k)$ numerically. Figures [6(a) and (b) show plots of $E_{\mathrm{AF}}(N, k)$ and $\xi_{\mathrm{SVNE}}$ versus $g$ for $N=4$ and $k=0,1,2$ in the case $\omega_{\mathrm{f}}=1.5, \omega_{\mathrm{a}}=1$. Avoided crossings occur at $g=0$, with a corresponding minimum in $\xi_{\text {SVNE }}$ that drops down to zero for each of the three states $\left|\phi_{4,0}\right\rangle,\left|\phi_{4,1}\right\rangle$ and $\left|\phi_{4,2}\right\rangle$. These states are therefore unentangled at $g=0$, i.e., in the absence of interaction between the two modes of the bipartite system, as one might expect. 

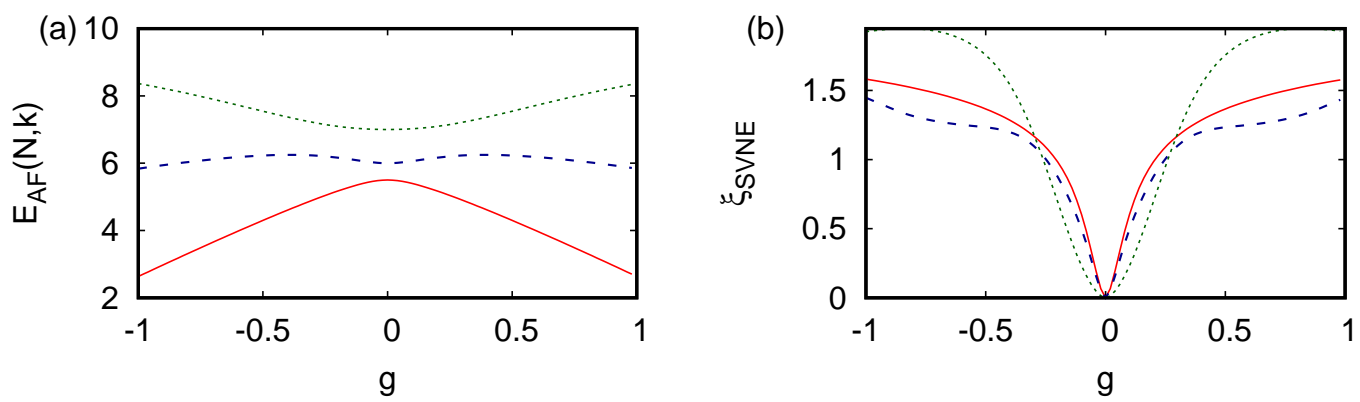

Figure 6. (a) $E_{\mathrm{AF}}(N, k)$ and (b) $\xi_{\mathrm{SVNE}}$ vs. $g$ for $N=4, k=0,1,2$ in the atom-field interaction model. The curves correspond to $k=0$ (red solid), 1 (blue dashed) and 2 (green dotted). $\omega_{\mathrm{f}}=1.5, \omega_{\mathrm{a}}=1, \gamma=1$.
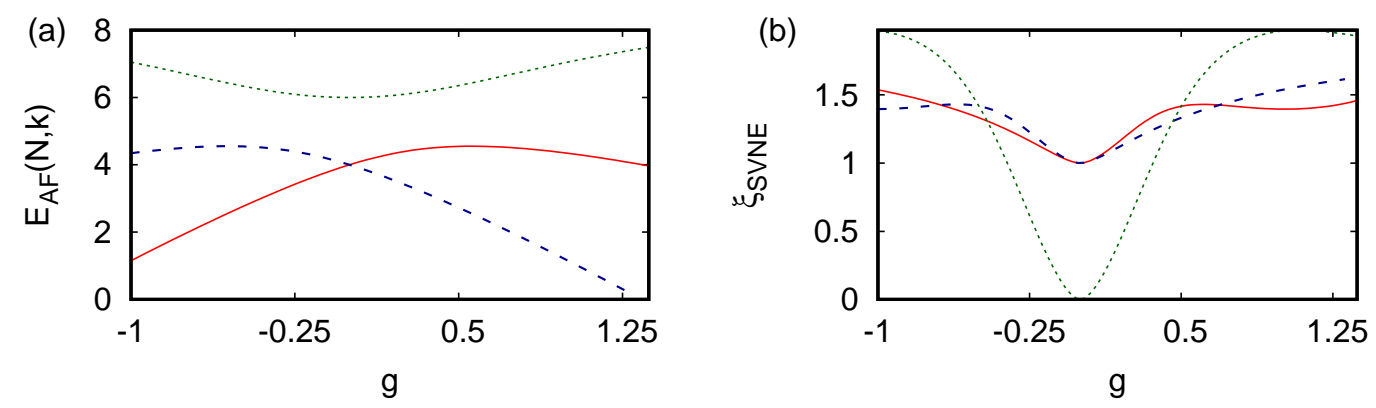

Figure 7. (a) $E_{\mathrm{AF}}(N, k)$ and (b) $\xi_{\mathrm{SVNE}}$ vs. $g$ for $N=4, k=0,1,2$ in the atom-field interaction model, in the degenerate case $\omega_{\mathrm{f}}=\omega_{\mathrm{a}}=1$. The curves correspond to $k=0$ (red solid), 1 (blue dashed) and 2 (green dotted). $\gamma=1$.

In order to examine what happens when there is a crossing of energy levels, we introduce a degeneracy by setting $\omega_{\mathrm{f}}=\omega_{\mathrm{a}}$. Figures 7 (a) and (b) are plots of $E_{\mathrm{AF}}(N, k)$ and $\xi_{\mathrm{SVNE}}$ versus $g$ for $N=4$ and $k=0,1,2$, with $\gamma, \omega_{\mathrm{a}}$ and $\omega_{\mathrm{f}}$ set equal to 1 . Both a level crossing and an avoided crossing are seen to occur at $g=0$, signalled by a minimum in $\xi_{\mathrm{SVNE}}$ for each of the three states concerned. The crossing of $E_{\mathrm{AF}}(4,0)$ and $E_{\mathrm{AF}}(4,1)$ arises as follows. Let $|p, 4-p\rangle$ denote the product state $|p\rangle \otimes|4-p\rangle$, where $|p\rangle$ is a photon number state of the field mode and $|4-p\rangle$ is an oscillator state of the atom mode. When $\gamma=\omega_{\mathrm{a}}=\omega_{\mathrm{f}}=1$ and $g=0$, the Hamiltonian reduces to $a^{\dagger} a+\left(b^{\dagger} b\right)^{2}$. The energy levels $E_{\mathrm{AF}}(4,0)$ and $E_{\mathrm{AF}}(4,1)$ become degenerate at the value 4 . The degeneracy occurs because the operator $|4,0\rangle\langle 3,1|+| 3,1\rangle\langle 4,0|$ commutes with $H_{\mathrm{AF}}$ when $\omega_{\mathrm{a}}=\omega_{\mathrm{f}}$ and $g=0$. Mixing of the states $|4,0\rangle$ and $|3,1\rangle$ occurs, and the corresponding energy eigenstates are given by the symmetric linear combination $\left|\phi_{4,0}\right\rangle=(|4,0\rangle+|3,1\rangle) / \sqrt{2}$ and the antisymmetric linear combination $\left|\phi_{4,1}\right\rangle=(|4,0\rangle-|3,1\rangle) / \sqrt{2}$. As the symmetries of the two states are different, the level crossing does not violate the von NeumannWigner no-crossing theorem. At the crossing, each of the states $\left|\phi_{4,0}\right\rangle$ and $\left|\phi_{4,1}\right\rangle$ remains a manifestly entangled state that is, in fact, a Bell state. This is why the corresponding $\xi_{\mathrm{SVNE}}$ does not vanish at that point, but merely dips to a local minimum with value 1, characteristic of a Bell state. It is interesting to note that the degeneracy that occurs 

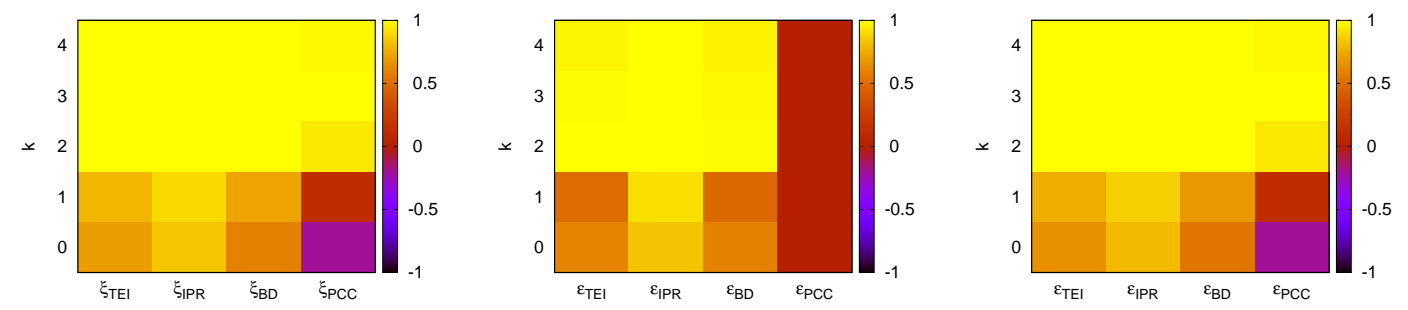

Figure 8. Correlation of $\xi_{\mathrm{SVNE}}$ with $\xi$-indicators (left), with $\varepsilon$-indicators for the slice $\theta_{\mathrm{A}}=0, \theta_{\mathrm{B}}=\frac{1}{2} \pi$ (centre), and with $\varepsilon$-indicators for the slice $\theta_{\mathrm{A}}=0, \theta_{\mathrm{B}}=0$ (right), for the eigenstates $\left|\phi_{4, k}\right\rangle, 0 \leqslant k \leqslant 4$ in the atom-field interaction model. $\omega_{\mathrm{f}}=\omega_{\mathrm{a}}=\gamma=1$.

when $\omega_{\mathrm{f}}=\omega_{\mathrm{a}}$ ensures entanglement even in the absence of any interaction between the two modes.

The level $E_{\mathrm{AF}}(4,2)$, on the other hand, is repelled and has the value 6 at $g=0$. The corresponding eigenstate $\left|\phi_{4,2}\right\rangle$ becomes the unentangled product state $|2,2\rangle$ at the avoided crossing, and $\xi_{\mathrm{SVNE}}$ drops to zero in this case, as expected.

In figure 8, we plot the correlation between various indicators and $\xi_{\mathrm{SVNE}}$. For this purpose, 80 values of each of the $\xi$-indicators were calculated with $g$ varied in the range $[-1,1.4]$ in steps of 0.03 . Treating these as sets of random numbers, we obtain the PCC between the various indicators and $\xi_{\mathrm{SVNE}}$, as described in the foregoing. The performance of the entanglement indicators in this case is similar to that found in the BEC system. Increasing $\gamma$ marginally decreases the efficacy of all the indicators.

\section{Avoided crossings in multipartite HQ systems}

\subsection{Tavis-Cummings model}

As our third and final example, we consider hybrid quantum systems comprising several qubits interacting with an external field. These systems are described by the class of Tavis-Cummings models [21] in a variety of diverse physical situations which include inherent field nonlinearities and inter-qubit interactions. The model we consider below is generic, applicable to a system of several two-level atoms with nearest-neighbour couplings interacting with an external radiation field in the presence of a Kerr-like nonlinearity, or to a chain of $M$ superconducting qubits interacting with a microwave field of frequency $\Omega_{\mathrm{f}}$. In the latter case, the model Hamiltonian (setting $\hbar=1$ ) is given by $[19,20]$

$$
\begin{aligned}
H_{\mathrm{TC}}=\Omega_{\mathrm{f}} a^{\dagger} a+\chi a^{\dagger 2} a^{2}+\sum_{p=1}^{M} & \Omega_{p} \sigma_{p z}+\Lambda\left(a^{\dagger} \sigma_{p}^{-}+a \sigma_{p}^{+}\right) \\
& +\sum_{p=1}^{M-1} \Lambda_{s}\left(\sigma_{p}^{-} \sigma_{(p+1)}^{+}+\sigma_{(p+1)}^{-} \sigma_{p}^{+}\right) .
\end{aligned}
$$

Here, $\chi$ is the strength of the field nonlinearity, $\Lambda$ is the coupling strength between the field and each of the $M$ qubits, $\sigma_{p}^{ \pm}$are the ladder operators of the $p^{\text {th }}$ qubit, and $\Lambda_{s}$ is 

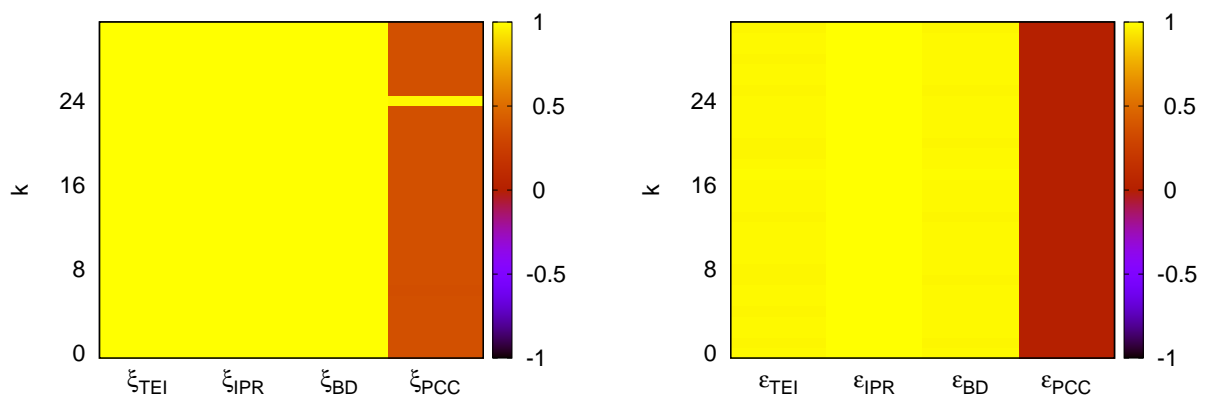

Figure 9. Correlation of $\xi_{\mathrm{SVNE}}$ with $\xi$-indicators (left) and with $\varepsilon$-indicators for the slice corresponding to $\theta=\frac{1}{2} \pi$ for the field and the $\sigma_{x}$ basis for each qubit (right). The figures are for the eigenstates $\left|\psi_{5,6, k}\right\rangle, 0 \leqslant k \leqslant 2^{5}-1$ in Case (i) in the Tavis-Cummings model.

the strength of the interaction between nearest-neighbour qubits. $\Omega_{p}=\left(\Delta_{p}^{2}+\epsilon^{2}\right)^{1 / 2}$ is the energy difference between the two levels of the $p^{\text {th }}$ qubit, where $\Delta_{p}$ is the inherent excitation gap and $\epsilon$ is the detuning of the external magnetic flux from the flux quantum $h /(2 e)$. In our numerical computations we have used the experimentally relevant [20] parameter values $\Omega_{\mathrm{f}} /(2 \pi)=7.78 \mathrm{GHz}$ and $\epsilon /(2 \pi)=4.62 \mathrm{GHz}$. The level separations $\Delta_{p}$ of the individual qubits have been drawn from a Gaussian distribution with a mean given by $\langle\Delta\rangle /(2 \pi)=5.6 \mathrm{GHz}$ and a standard deviation $0.2\langle\Delta\rangle$.

We have considered three cases, namely, (i) $\Lambda_{s}=\chi=0$ (ii) $\Lambda_{s} /(2 \pi)=1 \mathrm{MHz}, \chi=$ 0 (iii) $\Lambda_{s} /(2 \pi)=\chi /(2 \pi)=1 \mathrm{MHz}$. In each case, $\Lambda /(2 \pi)$ is varied from $-1.2 \mathrm{MHz}$ to $1.3 \mathrm{MHz}$ in steps of $0.025 \mathrm{MHz}$. It is easily shown that the total number operator

$$
\mathcal{N}_{\text {tot }}=a^{\dagger} a+\sum_{p=1}^{M} \sigma_{p}^{+} \sigma_{p}^{-},
$$

commutes with $H_{\mathrm{TC}}$. For each value of $\Lambda$ we have numerically solved for the complete set $\left\{\left|\psi_{M, N, k}\right\rangle\right\}$ of common eigenstates of $\mathcal{N}_{\text {tot }}$ and $H_{\text {TC }}$, where $N=0,1, \ldots$ is the eigenvalue of $\mathcal{N}_{\text {tot }}$ and $k=0,1, \ldots, 2^{M}-1$. Considering the total system as a bipartite composition of the field subsystem and a subsystem comprising all the qubits, we have computed the entanglement indicators. Figure 9 shows the correlation between the indicators and $\xi_{\mathrm{SVNE}}$ in Case (i). The associated Pearson correlation coefficients are 0.97 for $\varepsilon_{\mathrm{TEI}}, 0.99$ for $\varepsilon_{\mathrm{IPR}}, 0.97$ for $\varepsilon_{\mathrm{BD}}$, correct to two decimal places. (The accuracy of the $\varepsilon$-indicators depends, of course, on the basis chosen.) On averaging, we obtain the corresponding $\xi$-indicators with a PCC equal to 0.99 , showing that these indicators track $\xi_{\mathrm{SVNE}}$ very closely. We have carried out a similar exercise in Cases (ii) and (iii). The results and the inferences drawn from them are broadly similar to those found in Case (i).

Finally, with $\Lambda /(2 \pi)$ set equal to $1.2 \mathrm{MHz}$, we have examined the effect of changing the strength of the disorder in $\Omega_{p}$ by varying the standard deviation of $\Delta_{p}$ from 0 to $0.2\langle\Delta\rangle$ in steps of $2 \times 10^{-4}\langle\Delta\rangle$. Calculating the entanglement indicators for each disorder strength in $\Omega_{p}$, we have found the correlations between the $\xi$-indicators and $\xi_{\mathrm{SVNE}}$ in Cases (i), (ii), and (iii). $\xi_{\mathrm{TEI}}$ and $\xi_{\mathrm{BD}}$ turn out to be significantly closer to 
$\xi_{\mathrm{SVNE}}$, and hence more accurate indicators of entanglement, than the other indicators.

\section{Concluding remarks}

We have considered generic bipartite continuous-variable systems and hybrid quantum systems in the presence of nonlinearities, and tested quantitatively the efficacy of various indicators in estimating entanglement directly from quantum state tomograms close to avoided energy-level crossings. We find that the nonlinear correlation between the respective quadratures of the two subsystems reflects very reliably the extent of entanglement in bipartite CV systems governed by number-conserving Hamiltonians. We have shown that if the eigenstates of the Hamiltonian are Hamminguncorrelated, the inverse-participation-ratio-based quantifier $\xi_{\text {IPR }}$ is an excellent indicator of entanglement near avoided crossings. In fact, even $\varepsilon_{\mathrm{IPR}}$ (the corresponding indicator for a single section of the tomogram) suffices to estimate entanglement reliably. The tomographic entanglement indicator $\xi_{\text {TEI }}$ and the Bhattacharyya-distance-based indicator $\xi_{\mathrm{BD}}$ are also good indicators at avoided crossings, in contrast to the linear correlator $\xi_{\mathrm{PCC}}$ which is based on the Pearson correlation coefficient. Entanglement indicators seem to perform better with increasing $\left\langle N_{\text {tot }}\right\rangle$. The conclusions drawn are both significant and readily applicable in identifying optimal entanglement indicators that are easily obtained from tomograms, without employing state-reconstruction procedures.

\section{References}

[1] Nielsen M A and Chuang I L 2010 Quantum Computation and Quantum Information (Cambridge University Press, Cambridge)

[2] Paris M and Rehacek J 2004 Quantum State Estimation (Springer, Berlin)

[3] Sharmila B, Saumitran K, Lakshmibala S and Balakrishnan V 2017 J. Phys. B: At. Mol. Opt. 50 045501

[4] Sharmila B, Lakshmibala S and Balakrishnan V 2019 Quantum Inf. Process. 18236

[5] Sharmila B, Lakshmibala S and Balakrishnan V 2020 Quantum Inf. Process. 19127

[6] Vidal G, Latorre J I, Rico E and Kitaev A 2003 Phys. Rev. Lett. 90227902

[7] Karthik J, Sharma A and Lakshminarayan A 2007 Phys. Rev. A 75022304

[8] Oh S, Huang Z, Peskin U and Kais S 2008 Phys. Rev. A 78062106

[9] Bohigas O, Giannoni M J and Schmit C 1984 Phys. Rev. Lett. 521

[10] Haake F 2010 Quantum Signatures of Chaos (Springer, Berlin)

[11] Cejnar P and Jolie J 2000 Phys. Rev. E 616237

[12] Heiss W D 2012 J. Phys. A: Math. Theor. 45444016

[13] Pyzh M, Krnke S, Weitenberg C and Schmelcher P 2018 New J. Phys. 20015006

[14] Hutson J M, Tiesinga E and Julienne P S 2008 Phys. Rev. A 78052703

[15] Dembowski C, Gräf H D, Harney H L, Heine A, Heiss W D, Rehfeld H and Richter A 2001 Phys. Rev. Lett. 86787

[16] Barke I, Zheng F, Rügheimer T K and Himpsel F J 2006 Phys. Rev. Lett. 97226405

[17] Sanz L, Moussa M and Furuya K 2006 Ann. Phys. (N.Y.) 3211206

[18] Agarwal G S and Puri R R 1989 Phys. Rev. A 392969 
[19] Macha P, Oelsner G, Reiner J M, Marthaler M, André S, Schön G, Hübner U, Meyer H G, Il'ichev E and Ustinov A V 2014 Nat. Commun. 55146

[20] Shapiro D S, Macha P, Rubtsov A N and Ustinov A V 2015 Photonics 2449

[21] Tavis M and Cummings F W 1968 Phys. Rev. 170379

[22] Viola L and Brown W G 2007 J. Phys. A: Math. Theor. 408109

[23] Smith R 2015 Stat 4291

[24] Masada G and Furusawa A 2016 Nanophotonics 5469

[25] Cover T M and Thomas J A 2006 Elements of Information Theory (Wiley-Interscience, Hoboken)

[26] Kullback S and Leibler R A 1951 Ann. Math. Stat. 2279

[27] Bishop C 2006 Pattern Recognition and Machine Learning (Springer, New York)

[28] Kailath T 1967 IEEE T. Commun. Techn. 1552

[29] Thew R T, Nemoto K, White A G and Munro W J 2002 Phys. Rev. A 66012303

[30] Ibort A, Man'ko V I, Marmo G, Simoni A and Ventriglia F 2009 Phys. Scripta 79065013

[31] Trugenberger C A 2001 Phys. Rev. Lett. 87067901 